\title{
Kinematics of the orbital movement of a digital $X$-ray scanner of annular pipe seams
}

\author{
Rostislav Vasilchenko ${ }^{1}$, Fedor Simankin ${ }^{1}$, Grigorii Ziyakaev ${ }^{1}$, and Arkadiy Simankin ${ }^{2}$ \\ ${ }^{1}$ National Research Tomsk Polytechnic University, 634050 Tomsk, Russia \\ ${ }^{2}$ Tula State University, 300012 Tula, Russia
}

\begin{abstract}
The features of the kinematics of devices performing orbital displacements are considered. Different versions of the arrangement of mechanisms for the orbital movement of a digital radiographic scanner are compared. The load capacity of the support flexible joint of the mechanism was estimated.
\end{abstract}

\section{Introduction}

In ensuring the safety of operation of dangerous objects under pressure an important role is played by measures for non-destructive testing of the components dangerous objects [1-3]. In operation of pipeline systems under pressure and transporting corrosive liquid media, an important role is played by measures for non-destructive testing of the quality of welded joints of pipeline components. The existing methods of nondestructive testing are based on the study of the internal structure of materials and substances when they are exposed to radiographic (X-rays), infrared and gamma rays, ultrasonic vibrations, radio waves, magnetic and electric fields, penetrants, etc. Among other things, the radiographic method is more universal, since it allows testing the internal structure of the material and does not depend on climatic conditions. According to the standard [4, two types of sources are used to testing the annular weld seams: with a directional and panoramic output of Xray radiation. A directional outlet allows the pipeline to be inspected "through two pipe walls".

The scheme with a panoramic source located inside the object of testing and concentric to its central axis is simpler and more common. Panoramic X-ray machine makes it possible to scan through the film all the annular weld seams through one wall at once. Therefore, the main scope of work on quality testing of welded seams in the construction of pipelines is performed with panoramic X-ray generators with a self-propelled movement device called a "crawler". As a rule, classical (film) radiography is used with "crawlers". The essence of the method lies in the fact that an X-ray sensitive film is superimposed on the object of testing. Herewith seam material is located between the radiation source and the film. When the radiation is emitted, the film is illuminated non-uniformly, forming a shadow image of the structure of the controlled weld seam. In this way, the existing defects in welding are visible - fissures, cracks, caverns, etc. However, despite the seeming simplicity of the testing process, the features of the film development technology do not allow to evaluate quickly the quality of the received shadow image. In the event of abnormal situations, the seam testing process can be delayed for a long time. 


\section{Features of the kinematics of the orbital movement}

The ability to assess quickly the quality of the seam image and simplify the storage and processing of information on the monitored objects is provided by digital technologies. An example is the use of digital radiographic panels. In this case, in order to obtain a shadow image of the annular welded seam, it is necessary to conduct a scanning panel along the seam, i.e., the scanner movement mechanism should provide the orbital motion of the X-ray receiver relative to the monitoring object - a full revolution along the circular path, while the scanner's working surface is always directed to the center of the circle and perpendicular to its radius. To ensure a consistently high quality shadow image of the seam, the radiation receiver must perform a movement with a constant amount of distance from the radiator and from the object of the testing. To do this, the scanner must move around the object of testing, relying on the tube itself, while maintaining the scanner's constant position in the axial direction and a stable position in the radial direction with respect to the monitor object, regardless of the angle of rotation.

The sensory element is a scintillation detector. Such detectors make it possible to obtain shadow images of the internal structure of the material; however, they are very sensitive to focusing radiation. Thus, if the panoramic source is accurately positioned relative to the central axis of the pipe and the pipe meets the requirements for circularity, to ensure the required quality of the shadow image, it is sufficient to support the movable part of the scanner on the outer surface of the pipe or to a portable supporting surface specially installed on the pipe. As such a surface, a guide belt similar in design to those used for semi-automatic pipe welding by the CRC-Evans guide belts [5] can act as such a surface. In this case, the movement mechanism of the panel is pressed against the end surfaces of the belt by special rollers, one of which receives rotation from the drive with the electric motor and causes the scanner to perform orbital motion around the pipe with the object of testing [6]. However, the experience of operating such mechanisms (the semi-automatic welding head M300 or P600 of CRC-Evans [7]) indicates a high wear rate and, as a consequence, the high cost of the drive's leading rollers. In addition, belts are also susceptible to abrasion and should be replaced frequently.

There is another solution, which implements the orbital movement of the scanner around the object of testing. In this embodiment, the movable platform of the scanner is supported directly on the pipe. Herewith the platform is pressed against the pipe by a flexible joint (belt, chain, etc.). In addition to pressing, this joint serves as a guiding support element for the traction driver's unit. Any payload, such as a radiographic panel, batteries, electronic control units, etc., can be installed on the platform. In this design, the movement mechanism of the scanner can be equipped with different types of payload, which allows to take a shadow image of the weld seam with transmission through one or two walls of the pipe.

In general, to organize the orbital movement of a digital radiographic scanner, this layout looks more promising. The supporting elements do not experience increased loads; the design of such a mechanism is simpler. The mechanism does not need additional settings when installed on the pipe, except for adjusting the flexion force. The flexible joint itself is structurally simpler than the guide belt, it is more versatile when working with different nomenclature of the pipes being studied and advantageously differs in massdimensional characteristics. However, simplification of the construction of the support element (in our case, flexible coupling) can lead to a loss of strength characteristics, since in the process of work considerable tensile forces are applied to it. 


\section{Design scheme}

To evaluate the effort required for flexible communication, a design scheme was developed (Figure 1). This scheme allows to take into account the features of different layout elements on the scanner platform, quickly changing their location. For the numerical estimation of the load of flexible communication, a program was created in the MathCAD software.

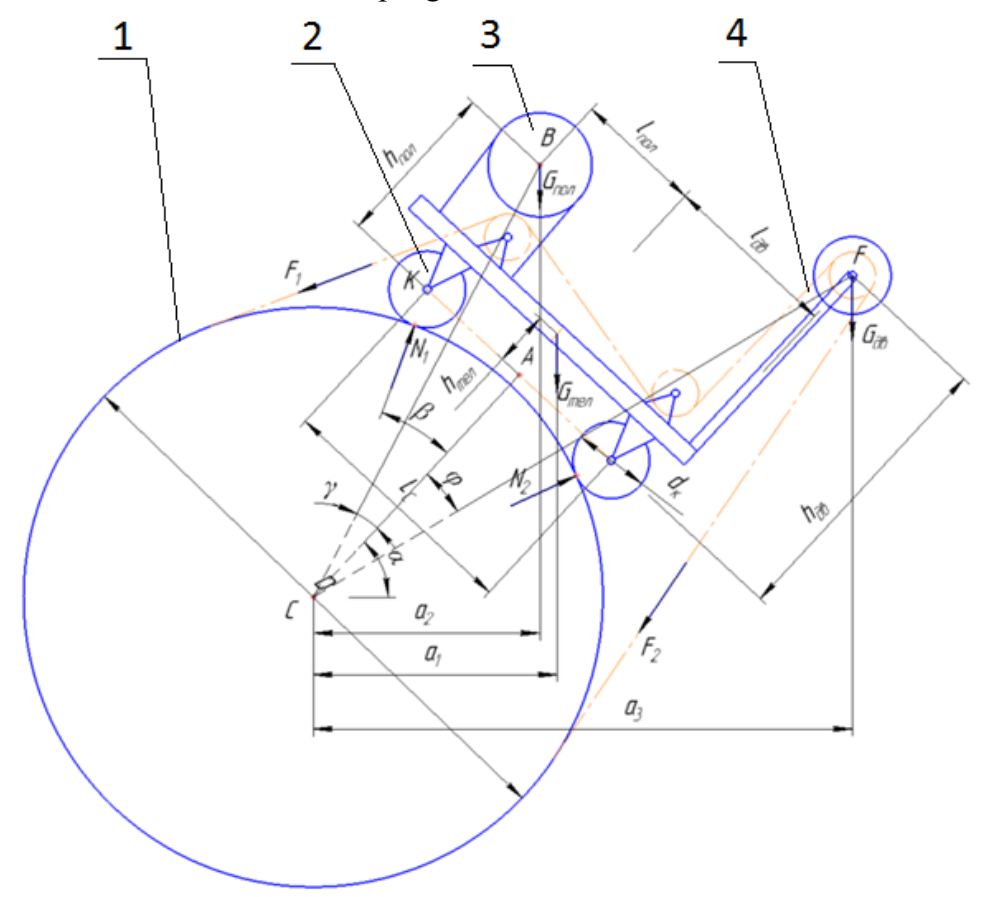

Fig. 1 Calculation scheme of the arrangement of the movement mechanism of the scanner of pipe seams: 1 - the object of testing (pipe with an annular welded seam), 2 - the platform with support wheels and the scanner drive, 3 - workload (scanner), 4 - flexible joint.

The results of the calculations (Figure 2) show forces stretching the flexible joint. The maximum forces arise on the section of the circular trajectory corresponding to the clockwise position in the range of 5 to 2 hours when moving clockwise. For example, with a payload of $40 \mathrm{~kg}$, the maximum forces is a little more than $4000 \mathrm{~N}$. Herewith and the torque that the scanner drive needs to keep the moving in this trajectory's section is 230 $\mathrm{N}^{*} \mathrm{~m}$. Such force values can be perceived without destruction by a flexible coupling made of metal or composite materials. To ensure flexibility, such joint must consist of separate elements that have mobility with respect to each other. 


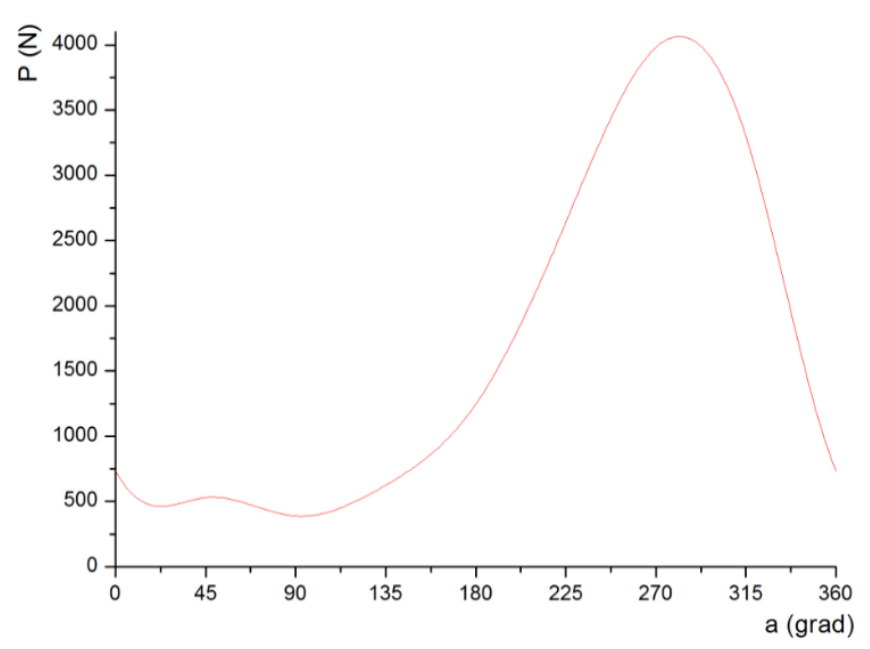

Fig. 2 The amount of tensile force applied to the flexible joint, depending on the angle of rotation of the mechanism around the pipe.

\section{Conclusions}

Thus, the considered arrangement of the mechanism for moving the X-ray scanner has a number of advantages over analogues using special guide belts for movement. In this complex "mechanism-object of testing" there is a flexible joint that ensures that the scanner is pressed to pipe and performs the function of a guiding support element for the traction driver's unit. To meet the conditions of strength in the process of working, this joint should be made of metallic materials. Structurally, such a joint can be made as a chain consisting of their individual strong elements, capable to swing relative to each other by a certain angle.

\section{References}

1. N. A. Ploskov, V. I. Danilov, L. B. Zuev, I. O. Bolotina, D. V. Orlova, Russian Physics Journal 54(12) (2012)

2. V. I. Danilov, L. B Zuev, I. O. Bolotina, A. A. Zagumennyi, Ph.of M. and Metal. 106 (3) (2008)

3. I. O. Bolotina, V. N. Borikov, V. S. Ivanova, K. V. Mertins, S. V. Uchaykin, J. of Petrol. Sc. and Eng. 161 (2018)

4. State standard 7512-82 «Non-destructive testing. Welded seams. Radiographic method»

5. Pipeline welding equipment.URL :

http://www.crc-evans.com/equipment/suppliessupport/band

6. X-ray scanner for weld seams.URL :

http://asgink.ru/userfiles/file/doc/Transcan-bucklet.PDF

7. Pipeline welding equipment.URL :

http://www.crc-evans.com/equipment/welding-machines/p-600 